\title{
Kommentar
}

Schweiz. Zschr. GanzheitsMedizin 17, 341-345 (2005). @ Verlag für GanzheitsMedizin, Basel. www.ganzheitsmedizin.ch

\section{Reimar Banis}

\section{Die tägliche Praxis der Psychosomatischen Energetik}

\author{
Ratschläge für eine erfolgreiche Anwendung
}

M ittlerweile etabliert sich die Psychosomatische Energetik (PSE) in immer mehr Praxen als Standardmethode. Das ist vor allem der Einfachheit und Schnelligkeit zu verdanken, mit der in wenigen Minuten ein präziser Überblick über das Energiesystem gewonnen werden kann, seelische Konflikte aufgespürt und mit geeigneten Komplexhomöopathika aufgelöst werden können. Dadurch kommt es zu Therapieerfolgen, die sich in Aussagen wie dieser wiederspiegeln:

«...Ich habe in mir und in einigen sehr eng vertrauten Menschen einen inneren Wandel festgestellt, den ich nie für möglich gehalten hätte. Die körperlichen Symptome - wegen derer die Menschen ja bekanntlich einen Arzt oder HP aufsuchen - schmolzen weg wie Eis in der Sonne - waren eigentlich nur zweitrangig - während sich ganz langsam und stetig die Ganzheit bildet.»

Das Interesse an der Erlernung der PSE steigt deshalb kontinuierlich, aber es gibt wenig Gelegenheit, praktische Informationen zu bekommen. Deshalb fragen mich immer wieder Kollegen zu Anfang ihrer Ausbildung, ob sich der Aufwand überhaupt lohnt, also einerseits für den Therapeuten, aber auch für seinen Patient, wie lange man für den einzelnen Patienten braucht, wieviel Honorar man verdient und wie die Behandlung konkret abläuft.

\section{Der Therapeut}

Zunächst möchte ich mich bei der Beantwortung dieser Fragen auf den
Behandler selbst konzentrieren. Wer ist geeignet, die Methode anzuwenden? Der Behandler sollte zunächst die Eingangskriterien der Zulassung erfüllen, also behördlich die Erlaubnis haben, Krankheiten zu diagnostizieren und zu therapieren. Dazu gehören Ärzte, Heilpraktiker und heilpraktisch tätige Psychotherapeuten, in der Schweiz ausgebildete Naturärzte. Die zweite Hürde, die schwieriger zu bewältigen ist, liegt in der Wesensnatur des Behandlers und seiner Vorbildung. Die besten Erfahrungen haben wir mit erfahrenen Therapeuten gemacht. Bei Anfängern habe ich oft den Verdacht, dass sie die PSE überschätzen und ihre Grenzen nicht genügend beachten.

Aspiranten der PSE sollten möglichst nicht zur Gruppe der sog. «StarTherapeuten» gehören. Wer nicht die nötige Demut aufbringen kann, sein Ego in den Hintergrund zu stellen und einfach zu sehen, was beim Energietest zum Vorschein kommt, der findet in der PSE nicht die für ihn passende Plattform. Der Behandler sollte genug Disziplin haben, das einfache, aber präzise anzuwendende Testschema zu befolgen. Auch sollte er am Anfang eigene Energieblockaden aufgelöst haben, um energetisch gesünder zu sein als seine Patienten, was die Qualität der Testergebnisse erheblich steigert. Man sollte der PSE auch einen genügend grossen Raum einräumen, denn sie mal eben zwischendurch zu machen, führt in der Regel nicht sehr weit. Daher gilt: entweder ganz oder gar nicht! Als Minimum sollte man zwei Halbtage während der Praxiswoche dafür reservieren, am besten morgens, wenn man frisch ist. Abends sind Energieteste in der Regel wesentlich störanfälliger.

Am Anfang genügt apparativ der einfache Basistestkasten, um erste Erfahrungen mit der PSE zu machen. Wichtig ist, an dieser Stelle nicht in eine Falle zu geraten, in die so mancher Anfänger hineingeraten ist und die mit der Anordnung und Eigenwilligkeit der Konflikte zu tun hat. Denn Konflikte können nach ihrer Anbehandlung oft für einige Wochen testmässig verschwinden. Der Arglose glaubt dann, der Konflikt sei geheilt. Vertrackterweise taucht oft zu allem Überfluss ein neuer Konflikt auf, dessen Therapie aber nicht anstehen sollte. Denn wir haben die Erfahrung gemacht, dass man einen Konflikt nach dem anderen auflösen sollte. Wenn man stattdessen ständig neue Konflikte anbehandelt, kann sich das gesamte psychoenergetische System destabilisieren. Wir haben schon Patienten erlebt, die innerhalb eines Jahres von ahnungslosen Kollegen acht oder sogar noch mehr unterschiedliche Konflikte behandelt bekommen haben und sich gar nicht besser fühlten, oft sogar schlechter, was aus unserer Sicht kein Wunder war. Es erübrigt sich zu sagen, dass solch ein Vorgehen die Methode in Misskredit bringt, ganz zu schweigen von der beschädigten Reputation des Behandlers.

Üblicherweise benötigt ein grosser Durchschnittskonflikt, den wir am häufigsten vorfinden, vier bis fünf Monate Therapie zu seiner Auflösung. Bestellt man den Patienten während dieser Zeit zu Kontrollen, kann man 


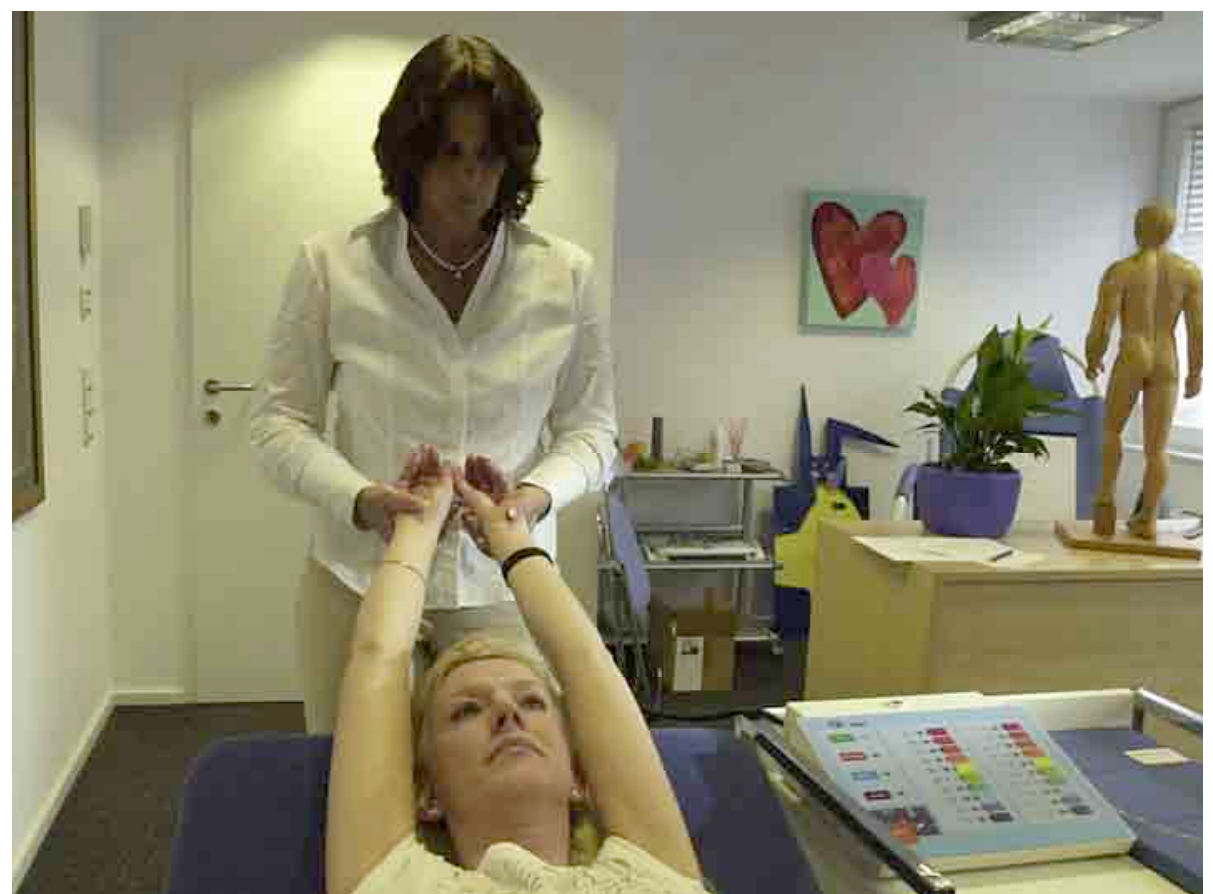

Typische Testsituation bei der Psychosomatischen Energetik - Armlängentest und Reba-Testung

kontinuierlich die Konfliktgrösse messen. Dazu benötigt der Anwender das Reba $^{\circledR}$-Testgerät, mit dem er auch einen Überblick über die Energiewerte seines Patienten erhält. Der Patient bekommt dadurch eine wichtige Information über den Heilprozess. Wenn man ohne das Gerät auskommen muss, also nur den Basistestkasten hat, sollte man den Patienten frühestens nach 8 Wochen nachtesten. Wenn man richtig testet, findet man sehr oft den gleichen Konflikt wie zuvor und weiss nun, dass dieser weiterbehandelt werden muss.

In puncto Gerät möchte ich erwähnen, daß wir immer wieder durch selbsternannte PSE-Therapeuten Negatives gehört haben, die ohne Vorbildung Sonderwege der PSE ersonnen haben oder sich sonstwie nicht regelkonform verhalten haben. Deshalb darf das Gerät neuerdings nur nach dem Besuch eines anerkannten Basisseminars verkauft werden. Den Besuch des Basisseminars halte ich für den Anfänger ohnehin für verpflichtend, genauso wie niemand auf die Idee käme, einen Blinddarm ohne Vorbildung zu operieren. Das psychoenergetische Gebiet verlangt jedoch mindestens genauso viel Vorbildung wie eine
Blinddarm-Operation!

Für den Test selbst rechnet man beim geübten Tester rund zehn Minuten, wobei wir wegen der Vor- und der ausführlichen Nachbesprechung eine Stunde pro Patient einplanen. Kontro luntersuchungen benötigen manchmal nur die Hälfte der Zeit. Der Patient braucht in der Regel keine besonderen Regeln beachten, sollte aber wenn möglich am Morgen der Untersuchung nur unverzichtbare Medikamente einnehmen, um klarere Testergebnisse zu bekommen. Am Testplatz sollte man ungestört arbeiten können und sich sicher sein, daß hier keine Geopathie und keine Elektrosmogbelastung vorliegt. Ich empfehle, Wireless Lan, DECT-Telefone ebenso wie Handys aus dem Testraum und seiner unmittelbaren Nachbarschaft zu verbannen oder zumindest auszuschalten. Angehörige sollten möglichst den Raum verlassen, es sei denn, bei kleinen Kindern geht es nicht anders.

\section{Der Patient}

Die nächste Frage ist, welche Patienten für den Test geeignet sind und welche nicht. Grundsätzlich dürfen Patienten skeptisch sein, sollten die grundsätzlichen Möglichkeiten der
Methode aber nicht zu sehr in Frage stellen, so dass daraus eine innere Blockadehaltung oder gar offene Opposition wird. Auch strikte Ablehner jedweder Psycho-Theorien haben wir schon geheilt, oder wenn wir den Konflikt im Gespräch überhaupt nicht erwähnt haben, so dass es sich bei der PSE im Unterschied zur Psychotherapie nicht um eine Methode handelt, wo der Patient die therapeutische Weltanschauung seines Behandlers teilen muss. Da die Untersuchung privat zu bezahlen ist, kommen ohnehin fast immer nur solche Patienten zur Untersuchung, die Dinge wie Homöopathie, feinstoffliche Energie, Erdstrahlen usw. anerkennen.

Eine weitere Bedingung zur erfolgreichen Anwendung der PSE kann man anfangs oft nicht voraussehen, dass der Patient nämlich genügend Disziplin aufbringen muss, um die Tropfen regelmässig einzunehmen. Bei fehlerhafter Mitarbeit des Patienten oder anderen Phänomenen, die zum Ausstieg auffordern, etwa überstarken Anfangsreaktionen, haben wir als erfahrene PSE-Anwender mit der Zeit den Eindruck gewonnen, dass daran sehr häufig die Konfliktaktivierung schuld ist, es sich also in Wahrheit um einen Widerstand des Konflikts handelt. Wenn der Patient dagegen die manchmal etwas stürmische Anfangsphase durchsteht, die meist nur eine Wochen dauert, selten länger, in der der Konflikt noch ein letztes Mal grossen Widerstand erzeugt, kommt es meist zu einer anhaltenden Bereitschaft, die Medikamente der PSE zu nehmen. Typische Patienten-Aussagen zu dieser Phase lauten: «Anfangs war ich skeptisch und fühlte mich sogar einige Zeit schlechter, meine Beschwerden waren sogar stärker, aber seitdem ich da durch bin, das dauerte einige Tage, geht es kontinuierlich aufwärts.»

Hierzu möchte ich noch anmerken, dass der grössere Teil aller Patienten wenig oder keine Verschlimmerungen verspürt. Man muss es als Behandler nur wissen, um nicht vorschnell zusammen mit seinem Patienten entmutigt zu sein. Nicht sinnvoll scheint es mir auch zu sein, die Tropfenzahl wochenlang reduziert einzunehmen, 
wie man immer wieder hören kann, weil der Patient dann in dieser heiklen Therapie-Phase nur wie in Zeitlupe vorankommt und sich nur unnötig quält. Er sollte stattdessen die benötigte Tropfenzahl, etwa 24 Tropfen täglich, in eine Flasche Wasser tun und schluckweise über den Tag verteilt trinken. Dann ist er viel schneller wieder in einem harmonischen Stadium, wo die Besserung rasch Fortschritte macht.

\section{Der Test}

Bei der Testung selbst kann jede energiemedizinische Testmöglichkeit benutzt werden, bei der sich der Untersucher sicher fühlt, sei es Kinesiologie, Einhandrute, EAV oder RAC. Wir empfehlen den kinesiologischen Armlängentest, weil er am wenigsten störanfällig ist und die sichersten Ergebnisse bringt. Wenn sauber und gut getestet wird, sind die Ergebnisse zuverlässig und unterschiedliche Untersucher kommen zu den gleichen Resultaten. Das gelingt aber nur in einer entspannten Atmosphäre ohne Mentalstress, so dass jede Befürchtung, man habe eventuell falsch getestet, beispielsweise bei wissenschaftlichen Doppelblindversuchen, zum Scheitern führen muss. Man sollte deshalb als Tester an sich glauben und in einer entspannten Weise vorgehen.

Der konkrete Testablauf wird im Basisseminar gelehrt. Auch wenn er anfangs leicht aussehen mag, stellt er doch hohe Anforderungen an die Testdisziplin und die Konzentrationsfähigkeit. Es gibt mittlerweile etliche regionale Arbeitsgruppen, wo erfahrene Tester die Methode unterrichten (siehe www.pse.name). Die Testung selbst dauert wie erwähnt nur wenige Minuten und viele Patienten sind überrascht, wie schnell der Test geht. Im Unterschied $\mathrm{zu}$ anderen Verfahren kommt es nicht so stark zur Ermüdung des Testers, trotzdem raten wir, nicht mehr als vier bis fünf Patienten hintereinander und am Tag höchstens acht Patienten zu testen. Dass man optimalerweise in den Tages-Hochphasen testen sollte, also von 8-12 und 14-16 Uhr, hatte ich bereits erwähnt.

Die Therapiekosten variieren von Patient zu Patient und hängen wesent- lich vom Krankheitsbild und der psychoenergetischen Gesamtsituation ab. Bei Kindern und einfach strukturierten Menschen, aber auch besonders Hochschwingenden mit grosser seelischer Offenheit genügt oft eine einzige Behandlung. Bei einer durchschnittlichen Konfliktgrösse dauert es minimal einen Monat, meist aber vier Monate zum Erreichen eines dauerhaften guten Zustandes. Wir empfehlen, mittels des Reba ${ }^{\circledR}$-Testgerätes zwischenzeitlich nachzusehen, wie stark der Konflikt abgeheilt ist. Erst wenn er so gut wie verschwunden ist, sollte man prüfen, ob ein neuer Konflikt anspricht. Oft hat es sich als ratsam erwiesen, dem Patienten gleich zu Anfang die Therapiemenge für vier Monate mitzugeben und ihn erst dann wiederzubestellen. Wer seine Patienten öfter sehen will oder muss, sollte sich auf das Messen der Konfliktgrösse und Überprüfen der Therapie beschränken.

Man sollte dem Patienten auch von Anfang an sagen, dass sich die Behandlung durchschnittlich über ein bis eineinhalb Jahre erstrecken kann, bis es zu einem zufriedenstellenden $\mathrm{Zu}$ stand kommt. Meist müssen zwei bis drei Konflikte aufgelöst werden, bis ein guter Therapieerfolg erreicht ist. In einer grossen Praxisstudie konnten wir bei $80 \%$ aller Patienten nach dieser Zeit einen guten bis sehr guten Behandlungserfolg erzielen. Wer nach einem Jahr der Behandlung noch keine wesentlichen Besserungen erlebt hat, gehört mit grosser Wahrscheinlichkeit zu den Non-Respondern, die es natürlich bei jeder Methode gibt. Es bleibt zukünftiger Forschung vorbehalten, hier durch Kombination mit anderen Verfahren und neuen Strategien weiterzukommen.

\section{Die Kosten}

Die Kosten liegen für den Patient bei durchschnittlich 50-150 € für die Erstkonsultation, je nach Zeitdauer und Qualifikation des Therapeuten. Kontrollen sind in der Regel preiswerter. Meist kommt man mit 4-5 Sitzungen aus, die sich über ein bis zwei Jahre erstrecken. In einigen Fällen reicht aber auch eine einzige Sitzung, etwa bei Kindern. Bei den Medikamenten rechnet man pro Sitzung etwa mit
8-12 Flaschen der Rubimed-Heilmittel, vorausgesetzt, man rezeptiert jeweils für 4 Monate im voraus. Bei Kindern reichen dagegen oft 1-2 Flaschen. Zusammengerechnet sind Gesamtkosten für eine Behandlungsserie von $600-1000 €$ realistisch.

Verglichen mit anderen vergleichbaren Verfahren kommt es zu einer vertretbaren und günstigen KostenNutzen-Relation. Die Therapie ist dann beendet, wenn der Patient gute Energiewerte hat, kein Konflikt mehr testet und sich der Patient wohlfühlt. Bei rund jedem vierten Patient eines durchschnittlichen Praxistages kann die Behandlung in dieser Weise erfolgreich beendet werden. Der Patient darf wiederkommen, wenn neue Beschwerden auftauchen oder wenn er das Bedürfnis nach einer Kontrolle äussert. Nicht wenige Patienten fühlen sich wohler, wenn sie hin und wieder zu einer Kontrolluntersuchung kommen dürfen. Insgesamt ist die Zufriedenheit mit der PSE sehr hoch und die Mundzu-Mund-Propaganda daher sehr verbreitet.

\section{Die Ausbildung}

Hinweisen möchte ich noch auf die Ausbildung zum «Zertifizierten Energietherapeuten» (das ist der Titel, der bei erfolgreichem Abschluss verliehen wird). Die gesamte Ausbildung erstreckt sich berufs begleitend über mehre re Jahre. Zunächst wird je ein eintägiges Basis- und Fortgeschrittenenseminar besucht. Dazwischen sollte mindestens ein Jahr liegen, um genug eigene Erfahrung zu sammeln. Inhalte des Basisseminars sind die grundlegenden Techniken der PSE, das Testen mit dem Basistestsatz und mit dem Reba ${ }^{\circledR}$ Testgerät. Zusätzlich werden auch die wesentlichen Konzepte der PSE vermittelt. Im Fortgeschrittenenseminar geht es um das weitläufige Thema des Zentralkonflikts, der mit dem eigenen Charaktertyp zu tun hat, sowie um das Arbeiten mit dem Organtestsatz.

Danach kann ein fünftägiges Intensivseminar besucht werden, an dessen Ende eine schriftliche und mündliche Prüfung zu bestehen ist, die zum Titel «Zertifizierter Energietherapeut» berechtigt. Wir versuchen, durch hohe Anforderungen auch nach bestande- 
ner Prüfung sowie durch intensive Weiterbildung ein möglichst hohes Niveau zu garantieren. Die gesamten Ausbildungskosten liegen bei den Seminargebühren unter 1000,- $€$. Nach der erfolgten Zertifizierung wird der Therapeut bevorzugt im Internet und bei Nachfragen von Patienten empfohlen. Jährlich finden für die Anwender der PSE zwei Treffen statt, bei denen man den aktuellen Stand der PSE erfahren kann, eine Vortragstagung bei der Medizinischen Woche BadenBaden und das zweitägige Expertentreffen. Die genaue Themenübersicht kann im Internet nachgelesen werden (unter www.rubimed.com).

\section{Erfahrung der KollegInnen}

Abschliessend möchte ich noch einige Erfahrungen und Bemerkungen zur Ermunterung zitieren, die Kollegen mit der PSE gemacht haben: «...Die
Patienten kommen sehr schnell an ihre entscheidenden unbewussten Probleme und lösen sie in kürzester Zeit und dauerhaft auf! Die erzielten Heilungen auch bei sonst therapieresistenten Fällen und die grossartigen spirituellen Fortschritte verblüffen mich tagtäglich aufs Neue! Es ist faszinierend, zu erleben, wie selbst Menschen, die zuvor von seelischen Belangen nicht viel wissen wollten, durch die Methode entscheidende Impulse zu mehr Offenheit erhalten, und sich charakterlich entwickeln.»

Eine andere Kollegin schreibt: «...Ich erfahre die Medikamente wie auch die gesamte Test-Methode immer wieder als segensreiche Hilfe für $\mathrm{Pa}$ tienten - und für mich als Therapeutin. Was war ich schon froh darüber, dass ich auf so einfache Art und Weise essentielle Blockaden und Organstörungen austesten konnte!!»

\section{Literatur:}

Banis R: Durch Energieheilung zu neuem Leben. Via Nova Verlag Petersberg 2002.

Banis R: Psychosomatische Energetik in der Allgemeinpraxis. Schweiz. Zschr. GanzheitsMedizin 2002;14(2):75-84.

Banis R: Lehrbuch der Psychosomatischen Energetik. Verlag VAK Kirchzarten 2003.

Banis R, Banis U: Psychosomatische Energetik Ergebnisse einer Praxisstudie, Schweizerische Zeitschrift für Ganzheitsmedizin 2004;16(3), 173-178.

Banis U: Naturheilkundliche Arbeit mit Kindern. Ermutigende Erfahrungen durch den Einsatz der Psychosomatischen Energetik. Schweiz. Zschr. GanzheitsMedizin 2002;14(7/8): 370-373.

Banis U: Praxis der Psychosomatischen Energ etik. Co'med Verlag Hochheim 3. Auflage 2005.

\section{Anschrift des Autors:}

Dr. med. Reimar Banis Facharzt für Allgemeinmedizin Grossmatt 3, CH 6052 Hergiswil drbanis@hotmail.com 